\title{
Reflets
}

Revue d'intervention sociale et communautaire

\section{Réflexion sur la place des hommes et les pères dans les services sociaux et de santé}

\section{Entrevue avec Gilles Rondeau}

\section{Jean-Martin Deslauriers}

Volume 15, numéro 1, 2009

Paternités : enjeux et perspectives (Première partie)

URI : https://id.erudit.org/iderudit/029585ar

DOI : https://doi.org/10.7202/029585ar

Aller au sommaire du numéro

Éditeur(s)

Reflets : Revue d'intervention sociale et communautaire

ISSN

1203-4576 (imprimé)

1712-8498 (numérique)

Découvrir la revue

Citer ce document

Deslauriers, J.-M. (2009). Réflexion sur la place des hommes et les pères dans les services sociaux et de santé : entrevue avec Gilles Rondeau. Reflets, 15(1), 20-35. https://doi.org/10.7202/029585ar d'utilisation que vous pouvez consulter en ligne. 


\section{Réflexion sur la place des hommes et les pères dans les services sociaux et de santé}

\section{Entrevue avec Gilles Rondeau}

L'entrevue suivante a été réalisée par Jean-Martin Deslauriers. Le comité de travail en matière de prévention et d'aide aux hommes dont il est question dans cette entrevue a publié, en 2004, un rapport intitulé Les hommes : s'ouvrir à leurs réalités et répondre à leurs besoins. Celui-ci a été remis au ministère de la Santé et des Services sociaux du Québec. Il contient seize recommandations touchant notamment les soins et les services pour les hommes, la paternité et l'identité masculine.

Reflets :

G. Rondeau :

On ne peut dissocier la réflexion sur les hommes de la question de la paternité. Dans le cadre des travaux du Comité de travail en matière de prévention et d'aide aux hommes, vous avez réuni des acteurs adhérant à différentes écoles de pensée concernant la situation des hommes. Quelles sont les divergences et convergences que vous avez observées parmi les acteurs qui s'intéressent à la question des hommes et de la paternité?

Avant que le ministre de la Santé et des Services sociaux donne le feu vert à la formation, au sein de son ministère, d'un comité pour étudier la question des hommes et des services leur étant offerts, un petit groupe de personnes s'était déjà constitué et avait fait pression auprès du ministre pour l'inciter à créer un tel comité. Ce premier groupe, dont je faisais partie, a reçu le mandat de recruter d'autres membres pour constituer un comité de travail. Notre intention était moins de sélectionner des personnes de différentes écoles de pensées que de regrouper des individus provenant de différents secteurs d'intervention auprès des hommes. Le comité 
a ainsi été composé de personnes qui connaissaient l'intervention auprès des hommes et qui œuvraient dans différents domaines, dont ceux de la violence, des groupes d'aide et de soutien et des problématiques liées au suicide. Nous nous sommes aussi assurés d'avoir une représentation régionale. Les questions relatives aux écoles de pensées étaient donc moins importantes que celles pertinentes à la connaissance des enjeux et des dossiers, à l'intérêt et à la disponibilité, à la diversité des expériences et à la provenance. Le comité comprenait, par exemple, un organisateur communautaire, des gens ayant milité de différentes façons pour la cause des hommes. Mais on n'y retrouvait aucune personne faisant partie de groupes identifiés comme radicaux ou extrémistes. Nous voulions un comité de travail actif et nous avons opté pour une approche pragmatique dans le but de faire avancer les choses. Nous avons conséquemment mis l'accent davantage sur ce qui nous réunissait et sur l'identification des éléments que nous estimions prioritaires. La paternité a rapidement été identifiée comme un élément central. Nous nous sommes entendus au départ pour dire que les hommes ne devaient pas être considérés comme étant des êtres "toxiques ", et nous avons adopté une approche prenant en compte autant la dimension de la prévention que l'angle curatif, nous interrogeant sur les besoins des hommes et sur le fait qu'ils aient pu être négligés par le système. C'est à partir de ces éléments de base que nous avons entrepris de réaliser le mandat qui nous avait été confié, celui de faire une évaluation de la situation des hommes en matière de santé et de services sociaux, d'identifier des éléments de réponse et de voir quels étaient les remèdes ou les recommandations qui s'imposaient.

Reflets :

Par la suite, quelles ont été les différentes réactions face à ce rapport?

G. Rondeau :

L'accueil a été généralement très positif. C'était la première fois qu'un rapport faisait état de toute une série de problématiques vécues par les hommes et présentait un portrait global de leur situation. Toutefois, et cela m'a beaucoup déçu, quelques mois après la publication du rapport, nous avons reçu des critiques du mouvement féministe qui, sur le plan du contenu, remettait en question la légitimité de la problématique soulevée et qui en 
Reflets :

G. Rondeau : rajoutait en dénonçant, disait-on, notre manque de sérieux et de rigueur. Ce sont essentiellement les seules critiques que nous avons reçues, hormis celles provenant des hommes proféministes radicaux. Dans les milieux de l'intervention, à la fois institutionnels et communautaires, le rapport a été très bien accueilli. Tous les groupes qui s'occupent des hommes l'ont salué avec beaucoup d'empressement, soulignant que nous avions tracé un portrait réaliste et nommé les vrais enjeux. Certaines personnes qui s'occupaient plus particulièrement des droits des hommes et qui étaient très sceptiques avant la publication du rapport, nous reprochant d'être vendus au féminisme, ont reconnu la pertinence du document et ont exprimé leur satisfaction face à son contenu. Nous n'avons pas fait notre travail dans un positionnement contre le mouvement des femmes, mais plutôt pour qu'il génère des retombées positives pour les hommes. Les membres de notre groupe croient que le féminisme est essentiellement bon et qu'il a apporté une importante contribution au développement de notre société. Notre rapport le souligne. Nous avons donc été déçus des réactions négatives du mouvement féministe. Par contre, malgré les points de vue négatifs exprimés par l'establishment féministe et par certains radicaux proféministes le rapport a été, de façon générale, très bien reçu. Et les idées qu'il contient sont toujours vivantes. Ce rapport est le début de quelque chose auquel il doit y avoir des suites.

Pouvez-vous préciser un peu plus les remarques qu'on vous a faites au sujet du rapport?

Essentiellement, on a dit qu'on créait un problème avec quelque chose qui n'en était pas un. Si je paraphrase, on m'a plus ou moins dit ce qui suit : "Y a pas de problème avec les hommes, ce sont les femmes qui ont des problèmes. Les hommes eux n'en ont pas puisque ce sont eux qui ont le pouvoir. Ils sont dans une position pour opprimer, ils ne sont pas dans une position de vulnérabilité ". Les critiques ont soutenu qu'en plus de traiter d'un problème social qui n'était véritablement pas sérieux, nous blâmions les femmes d'être surreprésentées dans le système de services et de mal s'occuper des hommes. Les mêmes critiques 
ont aussi dénigré la valeur scientifique du rapport, nous accusant notamment de citer certains de nos propres travaux. Nous avons répondu en soulignant que nous avions fait le maximum avec les maigres ressources qui nous avaient été allouées et que le rapport était suffisamment appuyé malgré tout. Même s'il s'agissait d'un rapport pour le gouvernement, on travaillait bénévolement et le budget était très limité. On doit comprendre que ce sont aussi les membres du comité qui ont assuré la rédaction, avec l'appui de deux fonctionnaires. Je me demande si ceux qui ont fait la critique n'ont pas eu plus d'argent que nous pour la rédiger. Je crois que notre rapport a en partie suscité une réaction du côté des critiques parce que nous réclamions de l'argent et des investissements pour davantage de ressources s'adressant aux hommes. Dans cette période de rareté, le mouvement féministe peut bien croire que l'argent qui pourrait être consacré aux ressources pour les hommes pourrait leur être enlevé. Ce n'était pas notre idée. Le rapport dit explicitement que nous ne voulons rien enlever aux femmes. Nous n'avons pas non plus demandé une symétrie sur le plan des services. Nous demandons simplement l'octroi de ressources pour les hommes qui ont des besoins et la prise en compte de ces mêmes besoins par un système qui pendant des années a négligé les services aux hommes. Je crois que les idées mises de l'avant dans notre rapport étaient présentes depuis un bon bout de temps, mais que, depuis l'avènement du féminisme, on n'en parlait pas parce que ce n'était peut-être pas bien vu d'en parler. Notre rapport rappelle qu'on est là, qu'on a des besoins. Lorsqu'on parlait des hommes récemment, c'était essentiellement pour dénoncer la violence, les actes criminels. Et mettre de l'avant les besoins des hommes, parler de leur vulnérabilité, de leur fragilité, ce n'était pas vu comme étant correct. Pas plus que de dire qu'il fallait s'en occuper.

En faisant notre travail de recherche, nous avons consulté les personnes qui sont dans le système, soit les intervenants qui sont dans les milieux de pratiques. Nous avons posé des questions, fait valider nos observations, sollicité le point de vue des gens impliqués auprès des hommes et demandé comment ils voyaient les problèmes. Notre rapport a reflété le point de vue des intervenants, 
Reflets:

G. Rondeau : des personnes sur le terrain, et c'est pour ça qu'il a été bien reçu. Selon moi, les idées sur le plan social évoluent beaucoup. On se rend compte qu'on ne peut pas soutenir un discours manichéen voulant que les hommes soient méchants et les femmes bonnes; que les hommes soient des agresseurs et les femmes de pauvres victimes, indépendamment des circonstances. Un tel discours ne reflète pas la réalité, car celle-ci est plus complexe. Sans que les choses soient simplifiées à un tel point, c'est comme si on laissait souvent entendre que ça s'apparente à ça. Nous n'avons pas rédigé un rapport idéologique, ou un rapport contre les femmes ou contre le mouvement des femmes. Nous avons voulu rédiger un rapport centré sur les faits. Plusieurs hommes sont prisonniers du même système qui fait aussi que les femmes sont infériorisées et victimes dans beaucoup de circonstances. C'est ce que nous cherchons à dénoncer.

De quelles façons trouvez-vous que les hommes et les femmes sont prisonniers d'un même système et que leurs conditions sont liées?

C'est la remise en question de leur situation par les femmes qui a donné lieu au féminisme. C'est ce même questionnement qui amène les hommes à s'interroger sur leur propre situation. Ainsi, alors que les femmes peuvent dire : « Le patriarcat nous maintient dans un état d'infériorité ", les hommes eux, peuvent soutenir : "Hormis les P.D.G., les gens de la classe gouvernante, ceux qui constituent le commun des mortels, hommes comme femmes, sont coincés dans un système où d'une certaine façon, ils vivent de façon semblable les exigences et contraintes de la domination. Nous devons nous questionner sur les gains à vouloir être toujours performants, à vouloir être toujours parfaits, supérieurs, à vouloir gagner à tout prix ". C'est cela que nous remettons en question. Notre projet de changement est très proche de celui des femmes. Nous appuyons ainsi le féminisme, et en même temps, le considérons comme une philosophie idéologique orientée vers le changement social. C'est bon aussi pour les gars parce que les stéréotypes masculins nous placent dans un univers que nous récusons. Et c'est bon pour tout le monde que de travailler à changer cela. 
Reflets :

G. Rondeau :
Quelle place est faite aux pères dans les services sociaux et de la santé?

Cela dépend comment on considère la question et aussi de quels services sociaux on parle. Notre étude de la situation nous a permis de constater qu'il existe peu de services pour les pères, tant dans le réseau de santé officiel que dans le réseau communautaire. Cependant, on observe que de plus en plus, les gens sont d'accord sur l'importance de développer des services sociaux et de santé s'adressant aux pères et aux hommes. On constate qu'il y a de plus en plus de préoccupations. Les suites à donner aux recommandations du rapport ont été confiées à un fonctionnaire du ministère de la Santé et des Services sociaux. La personne qui a été engagée pour cette fonction faisait partie de notre comité à l'origine. Elle a développé un plan d'action et identifié des répondants dans chaque région afin de voir à l'application des recommandations. Reste que les mécanismes de mise en action du ministère sont longs, compliqués et prennent du temps à s'actualiser. Cela se passe alors que nos demandes arrivent à un moment où le gouvernement effectue une réforme de l'ensemble de ses structures en matière de santé et de services sociaux, soit à une période où tout le monde se cherche. Mais il reste que l'importance de travailler le dossier des hommes est reconnue à l'intérieur du ministère, tout comme l'est la nécessité pour le système de santé et des services sociaux de réviser ses façons de voir et de faire à l'endroit des hommes. Des sessions de formation ont déjà été données à travers la province. Les CSSS [Centre de santé et de services sociaux] se sentent concernés. On leur demande de faire des choses. Ils ne répondent peut-être pas tous en même temps, ni avec le même niveau d'intensité, mais on constate des progrès. J'ai, par exemple, reçu un appel de mon CSSS m'informant qu'on mettait sur pied un comité pour rejoindre les hommes et qu'on m'invitait à y participer. Cette initiative émanait semble-t-il du ministère, via l'Agence de santé de Montréal, vers chacun des CSSS. Il s'agit donc d'un début. Du côté communautaire, c'est encore très petit, mais il y a malheureusement si peu d'argent disponible pour la paternité. Dans ma région, Montréal, c'est Centraide qui finance les organismes qui travaillent dans le domaine de la paternité. Je pense qu'il le fait parce qu'il en reconnait la nécessité tout comme 
Reflets :

G. Rondeau :

Reflets : il reconnaît l'importance des questions liées à la paternité. Ces quelques exemples illustrent que la mentalité des intervenants change, qu'on essaie d'apprendre aux intervenants et intervenantes à travailler avec les hommes, à arrêter d'en avoir peur, à nommer le travail qui doit être fait avec eux. On commence à développer le réflexe qu'il importe de demander aux pères, qu'il est vital de savoir ce qu'ils pensent dans les domaines où ils sont concernés afin de prendre leur point de vue en considération et d'organiser des services en mesure de mieux répondre aux besoins des hommes, tels ouvrir à des heures où ils peuvent avoir accès aux services, embaucher des intervenants masculins. On constate toute une série de détails qui n'étaient pas là auparavant. Par contre, la place faite aux pères est-elle suffisante? Les pères qui vivent une séparation, ceux qui sont en situation de crise ont peu de ressources à leur disposition. Il s'ensuit que souvent, des groupes militants formés de pères séparés vont d'une certaine façon, remplir le vide.

Par quels moyens peut-on tenir compte des besoins des pères face aux services sociaux tout en préservant les préoccupations?

La première chose, c'est de considérer que toute personne a des droits par rapport aux services publics, que ceux-ci sont ouverts à toute la population. On ne parle pas d'un service pour les femmes ou pour les hommes. C'est un service autant pour les hommes que pour les femmes, prêt à accueillir tout le monde. Par rapport aux services dispensés par les organismes publics, cela signifie que les intervenants de ces établissements doivent démontrer une volonté de rejoindre les hommes et se montrer capables de les écouter, de les aider et de les soutenir. Si on est d'accord avec ces éléments-là et si on investit l'énergie nécessaire pour faire une place aux hommes, pour les accueillir, on peut faire beaucoup de chemin dans la bonne direction. Pour moi, il s'agit de la base.

Plus tôt, vous disiez qu'il y a une convergence à établir entre les résultats du rapport du comité et les objectifs qui sont visés par le mouvement des femmes. Pensez-vous qu'il y aussi certaines tensions entre un point de vue qui explique les besoins des hommes et le mouvement des femmes? 
G. Rondeau :

Reflets :

G. Rondeau :
Pour moi, c'est clair qu'il y a une incompatibilité avec un féminisme radical, qui dit que les hommes n'ont pas de besoins, pas de vulnérabilités, qu'ils sont, à des degrés divers, des oppresseurs alors que les femmes sont leurs victimes. Je me demande où est la place d'un gars dans ce genre de discours. Là, effectivement, il y a incompatibilité. Évidemment si quelqu'un dit : "Moi je suis intervenante ou intervenant pour les femmes et les gars qui sont là, je ne peux ou ne veux pas les reconnaître ", oui alors là on est rendu au-delà de la zone limite. Il faut être au moins être capable de percevoir les difficultés vécues par l'homme qui se présente, être sensible à ses vulnérabilités, ouvert à ses besoins et comprendre qu'on peut l'aider. Si on n'est pas prêt à appréhender l'homme de cette manière, il est bien évident qu'on n'arrivera à rien. L'arrimage possible repose sur la capacité d'accepter que des hommes soient vulnérables, qu'ils aient des besoins légitimes, et qu'on puisse faire quelque chose pour eux. Sans cette posture de base, peu importe le référent idéologique, ça ne peut pas marcher. Et si le féminisme radical exclut les hommes, ne peut les voir comme ayant des besoins, ne peut les voir autrement que d'une façon négative, on ne pourra pas réussir avec lui.

Si je me fais un peu provocateur, est-ce qu'on pourrait dire qu'un féminisme radical exclut les hommes, les pères, qu'un féminisme plus ferme les oublie?

À mon avis, lorsque le féminisme s'est imposé, vers la fin des années 70, il y avait en effet beaucoup de chemin à faire pour que les femmes atteignent l'égalité, qu'elles soient reconnues, qu'elles acquièrent des droits et prennent des forces. La grande mobilisation qui s'est produite était nécessaire et c'est bien. Sauf que, depuis lors, les hommes sont pour ainsi dire tombés dans l'oubli; ils ne sont pas apparus comme ayant des besoins prioritaires ou essentiels puisqu'ils étaient perçus comme pouvant se débrouiller seuls. Par ailleurs, du côté des gars, demander de l'aide, c'est un problème parce qu'ils disent n'avoir besoin de rien, n'osant pas défier les normes traditionnelles de la masculinité. Je ne pense pas que les services que nous avons aujourd'hui résultent de mauvaises intentions, sauf qu'en regardant les résultats actuels, il faut se 
Reflets :

G. Rondeau : demander ce qui arrive aux hommes. Et on continue de nous ramener qu'il faut s'occuper des femmes! Je veux bien, mais il faut aussi s'occuper des gars! À un moment donné,j'étais frappé par des statistiques mentionnant qu'un pourcentage important de femmes étaient abusées sexuellement. C'est vrai.Et il faut agir pour leur venir en aide. Toutefois, les statistiques révèlent aussi qu'un pourcentage moindre mais quand même important d'hommes étaient eux aussi abusés sexuellement. S'il faut s'occuper des femmes, il faut aussi s'occuper des gars. Ce n'est pas parce que le nombre de femmes est plus important que nous devons négliger la minorité des hommes tout autant victimes. Leurs besoins à ces hommes ont été occultés, et c'est ça qu'on a voulu dire dans le rapport. Je ne crois pas que le féminisme modéré soit d'emblée insensible aux gars. Je ne veux cependant pas que pour des raisons idéologiques, ou même stratégiques, on conclue qu'il n'y a rien à faire pour les gars. Nous vivons ensemble dans une société qui se doit d'être accueillante pour tous.

Parmi les critiques envers le rapport du comité que vous avez présidé, on dit qu'il s'agit de l'œuvre de masculinistes.

Le mot " masculinisme " prête beaucoup à confusion. Gilles Tremblay de l'École de service social de l'Université Laval a fait la distinction entre deux sens pouvant être attribués au terme et qu'on a malheureusement tendance à confondre. Le mot " masculinisme » fait référence bien souvent à des militants prêts à prendre toutes sortes de moyens pour faire connaître leurs revendications et qui se disent victimes des femmes. L'autre sens du terme, c'est celui, beaucoup plus large, attribué à des hommes préoccupés par la condition des hommes et par l'ensemble des problèmes qui leur sont spécifiques, voulant connaître davantage les hommes pour mieux agir. Cette dernière définition est connue et fait partie de la littérature, mais dans le langage populaire, le mot " masculinisme " semble pour l'instant plutôt associé à ce qui est extrême. Le terme féminisme n'a pas cette signification péjorative attribuée au mot masculinisme. Il serait important de faire les distinctions qui s'imposent et ne pas mettre tout le monde dans le même bateau. 
Reflets :

G. Rondeau :
Les préoccupations entourant la situation des pères ont émergé au cours des dernières années, puis certains groupes de défense des droits des pères ont intensifié leurs pressions, notamment avec l'apparition de militants qui soutiennent que les pères séparés subissent d'importantes injustices. Comment situez-vous ces groupes dans vos actions?

Je ne suis pas très à l'aise avec les moyens extrêmes employés pour attirer l'attention sur les besoins. Parfois, j'ai l'impression que certains gestes sont posés par des hommes qui ont beaucoup de rancœur, de hargne et je ne suis pas favorable à ça. Par contre, je reconnais qu'il y a véritablement un malaise. Dans le cadre d'une étude dirigée par Germain Dulac, des pères qui étaient passés par un des organismes pour pères séparés ont été interviewés. Certains d'entre eux avaient été très malmenés dans le processus judiciaire et ils s'étaient retrouvés dépossédés, perdants. On ne peut pas encourager des gestes de haine, des gestes de violence, de désespoir, mais je suis persuadé qu'il y a des injustices à corriger. Lorsqu'il y a deux belligérants, c'est difficile de dire que tout le mal se situe d'un côté, tout le bien de l'autre. Je fais confiance aux juges pour partager ces choses-là. Ce ne sont pas des choses faciles à déterminer et j'imagine qu'avec la multiplication des accusations, on tombe dans un univers polarisé dans lequel les choses peuvent prendre des allures extrêmes. Les disputes concernant la garde des enfants peuvent entraîner ce qui s'appelle l'aliénation parentale. Par ailleurs, on peut porter des accusations qui peuvent prendre des proportions considérables. Il ne fait pas de doute que certains hommes qui sont violents avec leur conjointe peuvent aussi l'être avec leurs enfants. Ensuite, ils peuvent se plaindre d'avoir perdu leurs droits. Mais ces cas demeurent rares et sont loin d'être la norme. Il faut aider davantage et beaucoup mieux les hommes qui se séparent, les soutenir pour qu'ils puissent traverser la crise émotionnelle qu'ils subissent, leur faciliter l'obtention des renseignements légaux et les soutenir pendant la période de changement et d'adaptation. Il faut s'assurer qu'ils ne perdent pas leurs enfants dans le processus, qu'ils ne s'en désintéressent pas et qu'ils restent des pères. La parentalité est un contrat permanent alors que la vie de couple, elle peut prendre fin. Faire en sorte que la parentalité se poursuive après la séparation du couple 
Reflets :

G. Rondeau :

Reflets :

G. Rondeau : constitue une priorité. Je suis sensible aux besoins des hommes et il faut davantage de documentation sur les difficultés qu'ils vivent lors d'une séparation. C'est une problématique qui a été insuffisamment étudiée.

Quelles sont les possibilités d'échapper à un affrontement continuel entre les organismes de défense des droits des pères et des associations prônant un féminisme radical? Comment créer des passerelles entre ces tenants d'idéologies très différentes?

Je ne crois ni à l'un ni à l'autre de ces extrêmes et je ne pense pas qu'ils puissent aboutir à des solutions acceptables. Ces deux visions mènent à la fermeture et nous cantonnent dans des luttes stériles. Le succès et l'espoir se situent dans le milieu, dans l'équilibre. Il faut, je crois, adopter une approche plus inclusive, plus ouverte aux deux côtés, axée sur le respect et l'avancement. C'est en obtenant des succès dans des petites entreprises qu'on progresse et qu'on finit tous par être gagnants. Les luttes idéologiques sont comme les luttes religieuses, elles nous situent dans des chemins balisés par le dogmatisme, la rigidité. Par contre, les extrêmes sont des avertissements qui servent à nous tenir éveillés. La vraie piste à développer se situe au centre. Hommes et femmes sont, pour la majorité, appelés à vivre ensemble, à élever des familles.Y parvenir pacifiquement, de façon gratifiante et épanouissante pour les uns et pour les autres nécessite le respect, une capacité d'ouverture et d'écoute de l'autre. À mon avis, il faut travailler dans ce sens-là, et approcher les choses aussi d'une façon assez pragmatique.

Comment se concrétisera ce milieu fait d'écoute et de respect? Y a-t-il certains sujets, certains enjeux où ce serait plus facile d'y parvenir? Est-ce qu'il $y$ a des voies qui sont plus faciles à ouvrir?

Je pense qu'on y arrive de plus en plus. Il existait plus de tensions il y a vingt ans qu'aujourd'hui entre les hommes et les femmes. C'était davantage polarisé. On sent une certaine lassitude face à ces luttes entre positions irréconciliables, à ces luttes dites « du tout ou rien ». On avance vers plus d'ouverture. Après les grands changements qu'a connus notre société dans un temps très rapide, on observe plus d'égalité entre les hommes et les femmes. Nous 
devons persister vers l'objectif de vivre véritablement une telle égalité, de donner une vraie chance égale aux deux sexes, même si cela occasionne des bouleversements dans les structures, dans l'organisation sociale et dans nos vies personnelles. Au quotidien, dans nos vies privées, les choses ont beaucoup changé depuis 30 ans sur le plan des rapports entre les hommes et les femmes. Je pense qu'on est rendus à une participation beaucoup plus égalitaire. Les hommes sont beaucoup plus impliqués qu'auparavant dans la vie familiale. Ils assument davantage et différemment leur rôle de père et sont plus présents. Les deux parents sont là et les deux sont importants. À mon avis, en privilégiant la paternité et un rôle parental plus égal où les deux parents interagissent avec l'enfant, on atteint de meilleurs résultats. Un des grands changements survenus, c'est qu'on voit des pères partout avec des enfants. On les rencontre au jeu, à l'épicerie, à la pharmacie, dans une variété de sphères de la vie. Les hommes partagent aussi plus qu'avant les tâches ménagères, même si les femmes soutiennent qu'ils n'en font pas autant qu'elles. C'est peut-être vrai, mais comparé à la situation antérieure, on enregistre une réelle progression. Les pères vont chercher les enfants à la garderie, ils préparent les lunchs le matin, etc. Même si chaque couple vit ce partage à sa façon, il n'y a pas beaucoup de situations dans les familles au Québec où l'homme assume toujours le modèle traditionnel d'autrefois. Oui, il y en a encore des pères qui se font servir, qui n'interviennent que pour punir l'enfant, qui se voient comme des pourvoyeurs sans plus. Mais en général, ce n'est plus comme ça bien souvent. À cause des changements de rôle, les femmes peuvent davantage compter sur les hommes dans les tâches quotidiennes, ce qui est normal puisque la très grande majorité d'entre elles travaillent. Et les pères ont aussi réalisé que la proximité vécue avec leurs enfants leur apportait du bien-être; qu'ils avaient eux aussi un apport essentiel à donner à l'enfant, et que leur contribution faisait une différence. C'est sur ce plan qu'il y a eu, selon moi, le plus de progrès significatifs.

Reflets: $\quad$ Vous disiez plus tôt que les choses avaient évolué. Qu'est-ce qui s'est dénoué comme tensions, en tenant compte de la polarisation plus grande des années 80 ? 
G. Rondeau :
Pendant toutes ces années-là, le changement survenait à une très grande rapidité et les modèles d'autrefois se perdaient. On parlait plus fort, on s'affrontait davantage et là, tranquillement, on a fini par se dire qu'il fallait changer ce mode de confrontation. Les services sociaux, à mon avis, ont toujours été un univers de femmes, puisque les hommes y constituent une minorité inférieure à $15 \%$. Forcément, avec l'arrivée du féminisme, les femmes se sont engagées et plusieurs d'entre elles ont adhéré à l'approche féministe. La clientèle étant majoritairement composée de femmes, les intervenantes en sont venues à travailler essentiellement auprès des femmes et pour celles-ci. Après un certain temps, on a commencé à se demander ce qu'il en était des hommes. Je constate aujourd'hui une ouverture de la part de nombreuses femmes intervenantes qui disent qu'effectivement, elles ont eu d'autres priorités dans le passé, mais que désormais, il importe de prendre en compte différentes problématiques qui touchent aux hommes. Je ne vois pas ça comme un affrontement. Il s'agit plutôt de dire : «Il va falloir qu'on apprenne à changer nos mentalités, à nous ouvrir, à nous sensibiliser, et peut-être à adoucir nos positions idéologiques aussi ». Il existe 8000 organismes pour femmes et il n'y en a presque aucun pour les hommes. Je comprends aussi que ce qui est acquis, le mouvement féministe ne veut pas le perdre. Ce n'est pas ce que je préconise non plus, mais il va tout de même falloir accepter qu'une place plus grande devra être faite pour les hommes. C'est ainsi que je vois l'évolution dans les services sociaux au cours des prochaines années. Il y a par ailleurs tout un chemin à faire dans les écoles de service social et dans les écoles professionnelles de toutes les disciplines d'aide. Il faudra travailler à ce qu'il y ait plus d'intervenants masculins. Du nombre déjà restreint d'hommes intervenants dans les services sociaux, on observe que très peu travaillent dans le domaine clinique. Ce n'est pas toujours parce que les gars cherchent des emplois de dirigeants ou d'administrateurs, mais aussi parce que peu de femmes postulent à ces postes. À mon avis, on doit travailler à avoir de plus en plus de femmes dirigeantes de même que des hommes intervenants en plus grand nombre. Ça nous prend des 
Reflets :

G. Rondeau :

hommes pour enseigner, pour travailler à la garderie, sur le terrain en travail social et dans toutes les autres disciplines de relation d'aide. C'est important pour que les jeunes puissent avoir des modèles masculins et, en même temps, que plusieurs intervenants soient sensibles aux réalités des garçons. Il nous faudrait peut-être avoir des programmes d'équité.

Vous suggérez des programmes de soutien à la formation dans des disciplines non traditionnelles pour les hommes?

En vérité, ce qu'on a réussi. c'est que des femmes entrent dans des métiers non traditionnels jadis réservés aux hommes. Par contre, on n'est jamais parvenus à l'opposé, à savoir que davantage d'hommes entrent dans des métiers traditionnellement réservés aux femmes. Au mieux, on compte autour de $10 \%$ d'infirmiers.

Reflets :

En fait, est-ce qu'on a essayé?

G. Rondeau :

Voilà justement, on n'a pas essayé. À mon avis, on devrait faire de la discrimination positive. Il faudrait ainsi qu'il y ait dans les écoles de service social, dans les départements de psychologie, des programmes au sein desquels certaines places seraient réservées pour les hommes.

Reflets :

G. Rondeau :

Quels sont les changements qui vous ont le plus étonné dans l'évolution de la situation des hommes?

Pour moi, ce sont des changements qui viennent de prises de conscience. Une des premières choses dont j'ai pris conscience quand j'ai commencé à m'occuper des hommes qui avaient des comportements violents, c'est que ces hommes-là avaient parfois des qualités autres. Ils n'étaient pas que des individus violents. J'ai aussi réalisé, grâce à une de mes étudiantes, que les pères des milieux défavorisés étaient souvent extrêmement motivés à assumer leur rôle parental auprès de leurs enfants en dépit des difficultés qui marquaient leur vie. Ça m’a beaucoup frappé. Cette première étudiante qui faisait son stage dans un quartier défavorisé a mis sur pied un groupe où les pères ont manifesté un enthousiasme incroyable, du fait qu'on s'occupait d'eux. Un autre changement marquant, que j'ai mentionné, touche la présence 
des pères qui sont de plus en plus visibles et présents partout. On voit des pères prendre une part active dans les activités de leurs enfants. L'autre jour, par exemple, je suis allé à mon CLSC et j'ai observé que dans la salle d'attente, il y avait davantage de pères avec leurs enfants que de mères. Un autre phénomène récent concerne les congés de paternité. Ça surprend plein de monde que nombre de pères prennent de tels congés maintenant qu'on leur en donne la chance et qu'on assortit des conditions favorables à ce congé. Alors, je me dis qu'il faut regarder le côté positif, continuer à encourager les pères et favoriser leur participation afin que l'évolution de leur situation comme hommes et comme pères continue à progresser.

Pour finir, à mon avis, ce qui est notable c'est que les enfants sont au cœur du projet des hommes et que ceux-ci, tout comme les femmes, accordent une très grande importance à leurs enfants. Dans la mesure où la conjointe est prête à laisser son conjoint assumer un plus grand rôle, celui-ci aura une présence plus grande auprès de l'enfant. Ce qui est primordial toutefois, c'est de préserver les contacts père-enfant lorsque survient une rupture dans la vie du couple et de nous assurer que les pères, plutôt que d'abandonner, puissent continuer à jouer leur rôle tout autant qu'avant. Dans les situations de séparation, et encore plus malheureusement dans celles qui s'enveniment, c'est justement autour des enfants que tout se joue. Bien sûr, il y a quelques cas extrêmes où on ne peut pas maintenir la présence du père auprès de l'enfant. Toutefois, plus on peut favoriser une telle présence, même partielle, plus des résultats positifs seront parmi les retombées. Dans son contact avec l'enfant, le père, même s'il s'agit d'un criminel endurci, générera du bon pour son enfant et pour lui-même.

Reflets :

G. Rondeau :
Pourriez-vous élaborer justement sur les services concernant les pères?

J'ai parlé tout à l'heure de l'importance de mieux connaitre les situations des hommes qui se séparent. Une autre de mes préoccupations, c'est la façon dont les stéréotypes masculins se reproduisent d'une génération à l'autre. Comment expliquer le phénomène et comment le contrer? Il faudrait examiner cette 
question davantage. On n'en sait pas encore beaucoup sur qui sont les hommes, comment ils se comportent et comment leur venir en aide. Il y a énormément de recherche à faire sur le sujet, autant de travail pour la nouvelle équipe de recherches, Masculinités et société, que nous avons réussi à mettre sur pied. J'espère qu'elle aura une vie riche et longue.J'ai mentionné l'importance du père, le partage des rôles, le rôle de père. Ce sont là, à mon avis. tous des domaines qu'il faudra étudier davantage. Sur le plan des services, ça m'apparait comme une évidence qu'il n'y a pas suffisamment de services pour les hommes en général et de façon particulière pour ceux qui se séparent, pour ceux qui sont en détresse ou en situation de crise, pour ceux qui sont ou qui ont été victimes d'agression sexuelle.

Reflets:

Je vous remercie, Monsieur Rondeau, de nous avoir accordé cette entrevue. 